\title{
Confrontation d'un modèle théorique en lubrification mixte avec une étude expérimentale du comportement au frottement d'une denture d'engrenage droit
}

\author{
Farid Belarifi $^{1, a}$, Jean Blouet ${ }^{2}$, Geneviève Inglebert $^{2}$ et Ali Benamar ${ }^{1}$ \\ 1 Laboratoire de recherche en Technologie de Fabrication Mécanique, Département de Mécanique, ENSET, BP 1523, \\ El M'Nouer, Oran, Algérie \\ 2 LISMMA-EA2336 groupe de tribologie, SUPMECA, 3 rue Fernand Hainaut, 93407 Saint-Ouen Cedex, France
}

Reçu le 25 mai 2006, accepté le 7 décembre 2006

\begin{abstract}
Résumé - Nous proposons dans notre article, la confrontation entre un modèle de lubrification mixte et une étude expérimentale sur le comportement au frottement lubrifié d'une denture d'engrenage droit, effectuée sur un tribomètre pion disque. La configuration de la machine ainsi que les éprouvettes ont été adaptées pour l'étude du contact linéaire hertzien cylindre sur plan. Les travaux ont été menés sur quatre lubrifiants de différentes viscosités avec trois niveaux de pression. Les résultats montrent la convergence partielle ou totale entre le modèle et l'étude expérimentale.
\end{abstract}

Mots clés : lubrification / engrenages / contact hertzien / cylindre plan / pion disque

\begin{abstract}
Confrontation between a mixed lubrication model and an experimental survey on the behaviour to the friction of spur gear teeth. We propose in our paper, a theoretical study of a mixed lubrication model confronted to an experimental survey of the behaviour in lubricated friction condition of spur gear teeth, on a pin disk tribometer. The configuration of the machine and the test pin were adapted for the survey of the Hertzian line contact cylinder on plan. Work was led on four lubricants of different viscosity submitted to three levels of pressure. The results show partial or total convergence between the model and the experimental study.
\end{abstract}

Key words: lubrication / gears / Hertzian contact / cylinder plan / pin disk

\section{Introduction}

Les engrenages sont probablement l'un des plus anciens modes de transmission de puissance et cependant leur graissage est un problème des plus discutés [1].

Sachant que les profils des dentures sont des courbes, on est en présence d'un contact linéaire hertzien, pour lequel les déformations élastiques sont indispensables au fonctionnement et s'accompagnent de pressions de contact très importantes [2]. Il existe une seconde difficulté qui se trouve dans la nature même du mouvement des dentures : roulement et glissement y sont combinés; de plus, la vitesse de glissement change de sens au cours de l'engrènement. De ce fait, l'obtention d'un film d'huile lubrifiant s'interposant entre les dentures et obéissant aux seules lois de l'hydrodynamique est difficile. Martin [3] fut le premier à s'intéresser au problème du film d'huile

\footnotetext{
a Auteur correspondant : belarifi_farid@yahoo.fr
}

dans le cas des engrenages; par la suite Grubin [4] et Dowson [5] proposèrent des solutions numériques pour la formation du film d'huile dans un contact linéaire hertzien lubrifié.

Un certain nombre d'auteurs [1], constatent que dans la majorité des cas les dentures d'engrenages travaillent en mode de lubrification mixte. Dans la littérature on retrouve un certain nombre d'approches [6-8] qui expliquent l'influence de l'état de surface sur la variation de la répartition de la charge en lubrification mixte. Johnson et al. [7] notèrent dans leur article que la charge appliquée entre deux surfaces rugueuses en contact lubrifié se décompose en deux efforts, l'un dû au lubrifiant, l'autre dû aux aspérités de l'état de surface. Le modèle de Greenwood et Williamson [9] est utilisé pour calculer l'effort dû aux aspérités et la théorie de l'élastohydrodynamique permet de calculer l'effort dû au film d'huile [10]. Robbe-Valloire et al. [11,12] introduisent le phénomène de l'élastoplasticité, et proposent 


\section{Nomenclature}

\begin{tabular}{|c|c|c|}
\hline$a:$ & Longueur du pion & {$[\mathrm{m}]$} \\
\hline$b$ & Largeur de contact hertzienne & {$[\mathrm{m}]$} \\
\hline$E:$ & Module de Young & {$[\mathrm{GPa}]$} \\
\hline$E *:$ & Module de Young équivalent & {$[\mathrm{MPa}]$} \\
\hline$F:$ & Résultante des forces de frottement & {$[\mathrm{N}]$} \\
\hline$f:$ & Coefficient de frottement mixte & {$[-]$} \\
\hline$f_{\mathrm{s}}:$ & Frottement dans le domaine plastique & {$[-]$} \\
\hline$h:$ & Hauteur du film d'huile & {$[\mathrm{m}]$} \\
\hline$h_{0}:$ & Hauteur initiale & {$[\mathrm{m}]$} \\
\hline$h_{\mathrm{C}}:$ & Hauteur du film d'huile au centre & {$[\mathrm{m}]$} \\
\hline$l:$ & Largeur du pion & {$[\mathrm{m}]$} \\
\hline$L:$ & Hauteur totale du pion & {$[\mathrm{m}]$} \\
\hline$n:$ & Nombre d'aspérités dans le contact & {$[-]$} \\
\hline$p:$ & Pression moyenne & {$[\mathrm{MPa}]$} \\
\hline$R a:$ & Rugosité moyenne & {$[\mathrm{m}]$} \\
\hline$R_{1}:$ & Rayon de courbure de la roue (1) & {$[\mathrm{m}]$} \\
\hline$R_{2}:$ & Rayon de courbure de la roue (2) & {$[\mathrm{m}]$} \\
\hline$R_{\mathrm{e}}:$ & Rayon équivalent & {$[\mathrm{m}]$} \\
\hline$S:$ & Fonction de distribution gaussienne & [ ] \\
\hline$S_{\mathrm{N}}:$ & Surface normale & {$\left[\mathrm{m}^{2}\right]$} \\
\hline$S_{\mathrm{H}}:$ & Surface hydrodynamique & {$\left[\mathrm{m}^{2}\right]$} \\
\hline$S_{\mathrm{R}}:$ & Surface due aux aspérités & {$\left[\mathrm{m}^{2}\right]$} \\
\hline$S:$ & Paramètre de Stribeck & {$[\mathrm{m}]$} \\
\hline$U:$ & Somme des vitesses de roulement & {$\left[\mathrm{m} \cdot \mathrm{s}^{-1}\right]$} \\
\hline$V_{\mathrm{r}}:$ & Vitesse de roulement & {$\left[\mathrm{m} \cdot \mathrm{s}^{-1}\right]$} \\
\hline$V_{\mathrm{g}}:$ & Vitesse de glissement & {$\left[\mathrm{m} \cdot \mathrm{s}^{-1}\right]$} \\
\hline$W:$ & Charge appliquée & {$[\mathrm{N}]$} \\
\hline$W_{1}:$ & Résultante des forces visqueuses & {$[\mathrm{N}]$} \\
\hline$W_{\mathrm{S}}:$ & Résultante des forces dues aux aspérités & {$[\mathrm{N}]$} \\
\hline \multicolumn{3}{|c|}{ Lettres grecques } \\
\hline$\alpha:$ & Angle de pression & {$[\mathrm{rd}]$} \\
\hline$\alpha:$ & Coefficient de piézoviscosité du lubrifiant & {$\left[\mathrm{Pa}^{-1}\right]$} \\
\hline$\beta:$ & Rayon de courbure de l'aspérité & {$[\mathrm{m}]$} \\
\hline$\delta:$ & Déformation & {$[\mathrm{m}]$} \\
\hline$\gamma:$ & Taux de cisaillement & {$\left[\mathrm{s}^{-1}\right]$} \\
\hline$\eta_{0}:$ & Viscosité dynamique & {$[$ Pas $]$} \\
\hline$\sigma:$ & Distribution gaussienne & {$[\mathrm{m}]$} \\
\hline$\varpi:$ & Vitesse angulaire & {$\left[\mathrm{rd} . \mathrm{s}^{-1}\right]$} \\
\hline$\tau:$ & Contrainte de cisaillement dans le lubrifiant & {$[\mathrm{MPa}]$} \\
\hline$\tau_{0}:$ & Contrainte de Eyring & {$[\mathrm{MPa}]$} \\
\hline
\end{tabular}

un modèle de lubrification mixte entre deux surfaces parallèles. Le modèle de Gelink [13] se base sur les travaux de Greenwood et Williamson [9] et extrapole le modèle de Johnson [7] pour représenter les courbes de Stribeck dans le cas du contact linéaire. Paffoni et al. [14] utilisent le modèle de Robbe-Valloire pour calculer numériquement le coefficient de frottement dans le cas d'un contact sphère sur plan lubrifié.

L'objectif de ce travail est de simuler expérimentalement le comportement au frottement lubrifié d'une denture droite en conditions extrêmes de frottement avec quatre lubrifiants de différentes viscosités soumis à trois niveaux de pression, pour une gamme de vitesses de glissement variant de 0,15 à $3 \mathrm{~m} . \mathrm{s}^{-1}$. Une confrontation des résultats avec un modèle se situant entre le modèle de Gelink [13] et celui de Paffoni [14] est proposé.

Les essais ont été effectués sur un tribomètre pion disque, adapté pour garantir le contact linéique, et par la suite tracer la courbe de frottement en fonction de la vitesse $V$, de la pression $p$ et de la viscosité du lubrifiant $\eta_{0}$, qui est nommée généralement la courbe de Stribeck. Cette courbe peut être décomposée en trois zones de lubrification qui sont le domaine de la lubrification limite, la lubrification hydrodynamique ou élastohydrodynamique et 


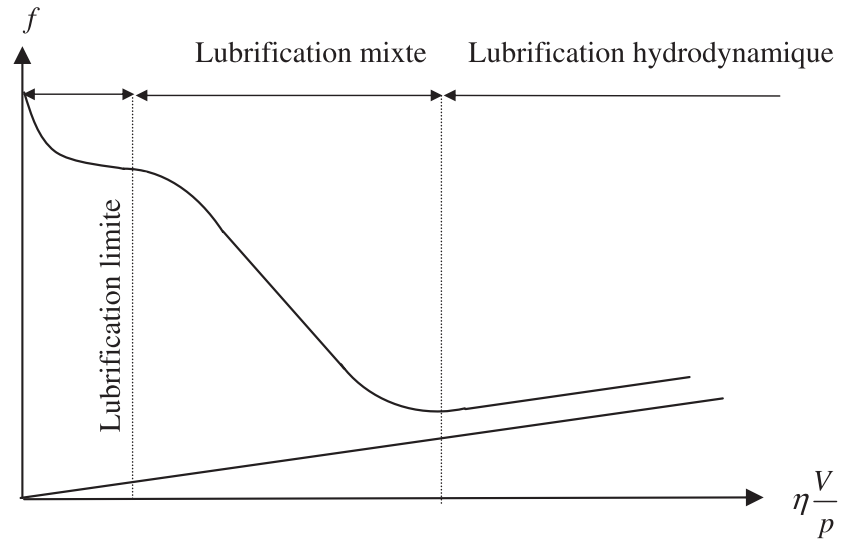

Fig. 1. Courbe de Stribeck.

la lubrification mixte qui est la transition entre les lubrifications limite et hydrodynamique, [15], la figure 1 indique la position de ces différents régimes sur la courbe de Stribeck. Cette courbe donne le coefficient de frottement global $(f)$ en fonction du paramètre $S$ qui est défini initialement par

$$
S=\eta_{0} \frac{V}{P}
$$

Un certain nombre de formulations ont été données depuis au paramètre $S$ pour prendre en compte des effets complémentaires liés à l'état de surface ou au matériau.

\section{Contexte de l'étude}

Ce travail s'inscrit dans le cadre de l'étude du comportement d'une denture droite en conditions extrêmes de frottement. En effet, toutes les études relatives aux engrenages se déroulent soit sur des bancs d'essais spéciaux [2], soit sur des machines bi-disques $[1,2]$, mais les informations concernant l'étude en conditions extrêmes de frottement en régime de glissement pur sont rares voire inexistantes. Or, c'est dans ces conditions que naissent les problèmes relatifs à la détérioration des dentures [1]. Un modèle cinématique de l'engrènement entre deux dents en prise dont on considère le contact en mouvement est représenté par la figure 2a [2]; l'entrée du contact est matérialisée par le point A et la sortie du contact par le point $\mathrm{B}$. On désigne par $R_{\mathrm{b} 1}$ le rayon de base pour le pignon (1), $R_{\mathrm{b} 2}$ le rayon de base de la roue (2), et par I le point d'engrènement sur le cercle primitif. On désigne par $\varpi_{1}$ et $\varpi_{2}$ les vitesses angulaires du pignon et de la roue, et par $\alpha$ l'angle de pression; l'analyse cinématique du mouvement lors de l'engrènement d'après Lefèvre [1], Henriot [2], montre que les flancs des dents ne roulent pas l'un sur l'autre sans glisser, et que la vitesse de roulement varie sur toute la longueur de contact (Fig. 2). Pour la formation du film d'huile on considère que [16] :

- La vitesse de roulement globale s'écrit:

$$
U=V r_{1}+V r_{2}
$$

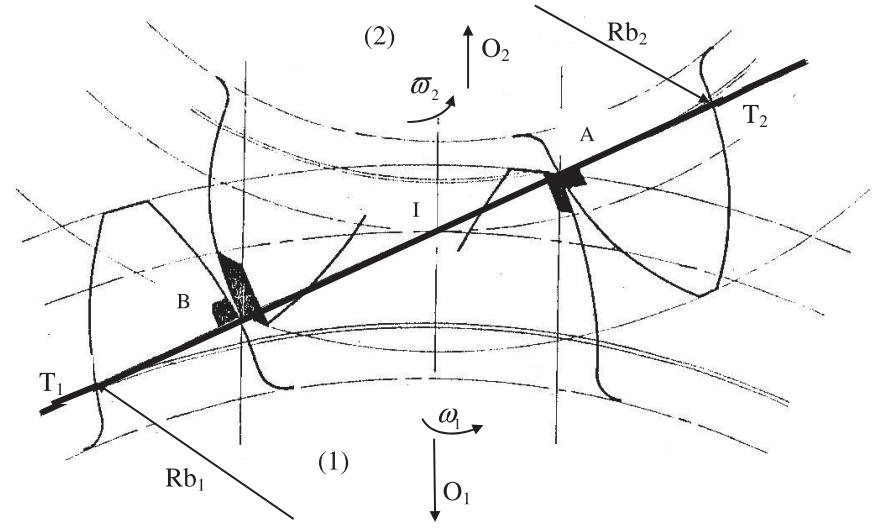

(a)

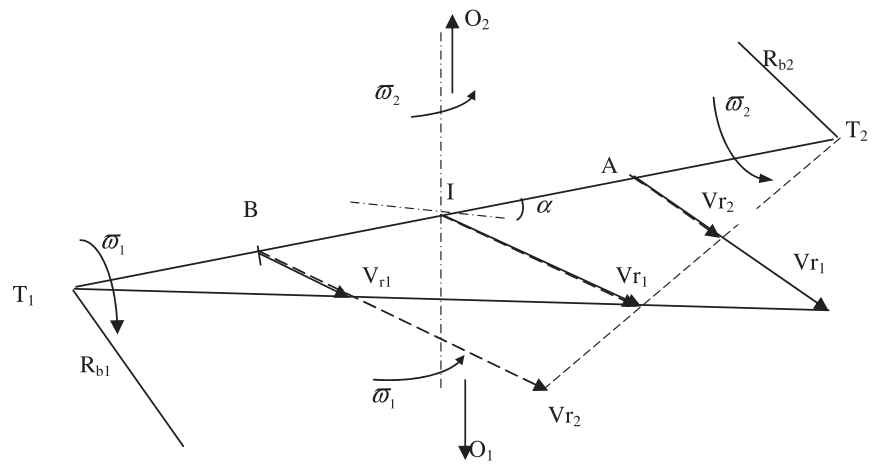

(b)

Fig. 2. a) Situation d'engrènement, b) situation cinématique.

- La vitesse de glissement s'écrit :

$$
V_{\mathrm{g}}=\left|V r_{1}-V r_{2}\right|
$$

- Le taux de glissement s'écrit :

$$
\lambda=\frac{V_{\mathrm{g}}}{U}
$$

Le bulletin 95 [16] de l'institut des engrenages et des transmissions indique que la vitesse de roulement globale augmente du point $T_{1}$ au point $T_{2}$, et la vitesse de glissement augmente du point $\mathrm{I}$ au point $\mathrm{T}_{1}$ et de $\mathrm{I}$ à $\mathrm{T}_{2}$. Quant au taux de glissement $[1,2,16]$, il s'annule au primitif au point I, où $V r_{1}=V r_{2}$, et prend la valeur de un en $\mathrm{T}_{1}$ et $\mathrm{T}_{2}$ où $V r_{1}$ et $V r_{2}$ s'annulent, figure $2 \mathrm{~b}$.

Les observations faites par les travaux de Henriot [2], Borsoff [17] et d'autres auteurs [1] montrent que l'usure des engrenages se fait dans les conditions extrêmes de frottement et se localise à l'entrée et à la sortie du contact. Notre étude se focalise sur le point A, pour lequel la vitesse de glissement est très grande. C'est une situation en conditions extrêmes de frottement qu'on peut simuler sur un tribomètre pion disque.

Le contact de deux dents en prise d'un couple d'engrenages se modélise géométriquement comme un contact entre deux cylindres ou deux demi-cylindres (Fig. 3a) de rayons $R_{1}$ et $R_{2}$ tournant à des vitesses $\varpi_{1}$ et $\varpi_{2}$. et dont 


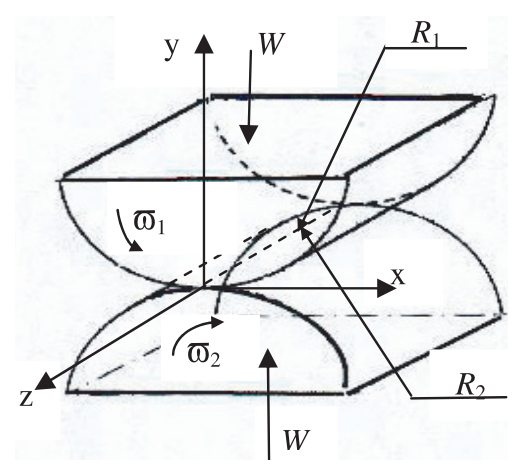

(a)

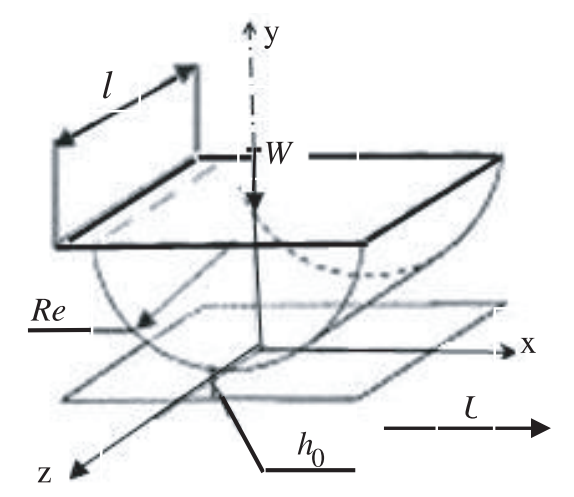

(b)

Fig. 3. a) Modèle de contact entre deux dentures, b) géométrie équivalente.

le contact cylindre sur plan représente un cas particulier (Fig. 3b).

\section{Modélisation du frottement en lubrification mixte}

La lubrification mixte est un régime intermédiaire entre la lubrification limite et la lubrification hydrodynamique figure 1, dans laquelle l'interaction entre les états de surfaces est mise en évidence. La compression de deux surfaces l'une contre l'autre sous l'effet d'une charge normale $W$ figure 4, l'une idéalement lisse, l'autre rugueuse, représente une situation à laquelle on peut toujours se ramener dans le cas de deux surfaces rugueuses selon le concept de Mc Cool [18].

On remarque d'après la figure 4 , que la charge $W$ se décompose en deux forces de types hydrodynamique de résultante $W_{\mathrm{PVR}}$, ou élastohydrodynamique $W_{\mathrm{EHD}}$, ces deux efforts engendrent des films d'huile de différentes hauteurs $h^{*}$ non nulles. La somme de ces efforts donne une portance d'origine visqueuse qui dépend entre autres de la vitesse de glissement et du lubrifiant; on peut l'écrire :

$$
W_{\mathrm{L}}=W_{\mathrm{PVR}}+W_{\mathrm{EHD}}
$$

Il en découle la force de frottement visqueuse

$$
F_{\mathrm{L}}=f_{\mathrm{L}} \cdot W_{\mathrm{L}}
$$
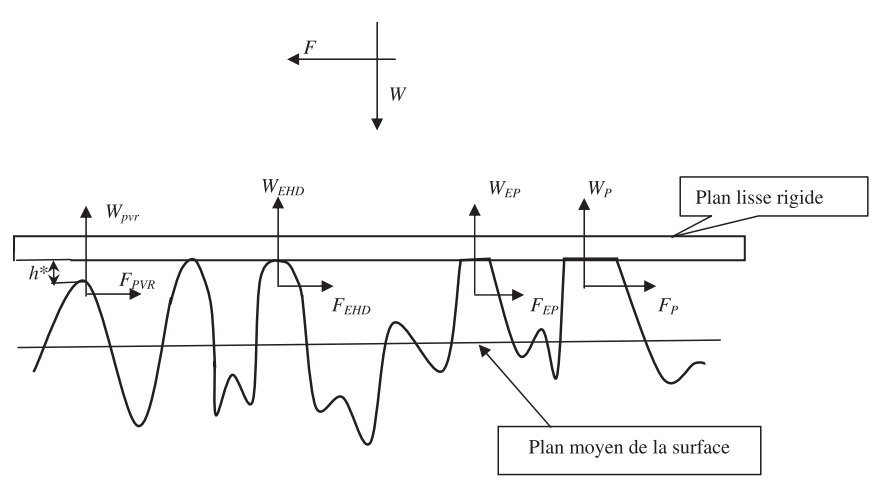

Fig. 4. Différents efforts supportés par les aspérités.

Les aspérités en contact avec la surface lisse sont soumises à un écrasement. Selon l'importance de cet écrasement qui peut être modéré ou important, on aura un effort élastoplastique dans le premier cas de résultante $W_{\mathrm{EP}}$ et plastique dans le second cas de résultante $W_{\mathrm{P}}$.

La somme des efforts donne une portance de type solide indépendante de la vitesse et du lubrifiant, qui s'écrit :

$$
W_{\mathrm{S}}=W_{\mathrm{EP}}+W_{\mathrm{P}}
$$

L'effort de frottement est de type solide qui dépend d'un coefficient de frottement global qui s'écrit :

$$
F_{\mathrm{S}}=f_{\mathrm{S}} \cdot W_{\mathrm{S}}
$$

La force normale que supporte le contact résulte d'une portance solide et d'une portance visqueuse et s'écrit :

$$
W=W_{\mathrm{L}}+W_{\mathrm{S}}
$$

La force de frottement globale s'écrit :

$$
F=F_{\mathrm{L}}+F_{\mathrm{S}}=f_{\mathrm{L}} \cdot W_{\mathrm{L}}+f_{\mathrm{S}} \cdot W_{\mathrm{S}}
$$

Le coefficient de frottement global s'écrit :

$$
f=\frac{F}{W}
$$

Pour simplifier les calculs on considère dans un contact hertzien que toutes les aspérités se trouvent dans un état de lubrification élastohydrodynamique donc $W_{\mathrm{PVR}}=0$,

D'où l'équation (5) qui devient :

$$
W_{\mathrm{L}}=W_{\mathrm{EHD}}
$$

En faisant intervenir les contraintes de cisaillement et le comportement rhéologique du lubrifiant [19] la force de frottement visqueuse s'écrit :

$$
F_{\mathrm{EHD}}=F_{\mathrm{L}}=\int_{S} \int_{\mathrm{H}} \tau_{\mathrm{H}}(\gamma) \mathrm{d} S_{\mathrm{H}}
$$

En introduisant le modèle de Eyring [10, 13, 19] dans l'équation (12), on obtient :

$$
F_{\mathrm{EHD}}=\tau_{0} \cdot\left(S_{\mathrm{H}}\right) \operatorname{ArcSinh}\left(\frac{\eta \cdot V_{\mathrm{g}}}{h_{\mathrm{c}} \tau_{0}}\right)
$$


Dans les conditions isothermes on a $\eta=\eta_{0}$.

La surface hydrodynamique s'écrit :

$$
S_{\mathrm{H}}=S_{\mathrm{N}}-S_{\mathrm{R}}
$$

où $S_{\mathrm{N}}$ représente la surface due à la pression hertzienne

$$
S_{\mathrm{N}}=\pi . l . b
$$

et $S_{\mathrm{R}}$ représente la surface réelle due aux aspérités et se calcule par le modèle de Greenwood et Williamson $[9,10,13]$ dont l'équation est :

$$
S_{\mathrm{R}}=\pi n \beta\left(S_{\mathrm{N}}\right)\left(\sigma_{\mathrm{s}}\right) F_{1}\left(\frac{h^{*}}{\sigma}\right)
$$

Sachant que pour une distribution gaussienne on a : $[7,9,13]$

$$
\begin{gathered}
\sigma=1,25 R_{\mathrm{a}} \\
\sigma_{\mathrm{s}}=0,71 \sigma
\end{gathered}
$$

La fonction $F_{1}\left(\frac{h^{*}}{\sigma}\right)$ s'écrit en fonction de la fonction de distribution gaussienne $s$ :

$$
F_{1}\left(\frac{h^{*}}{\sigma}\right)=\int_{\frac{h^{*}}{\sigma}}^{+\infty} \frac{1}{\sqrt{2 \pi}}\left(s-\frac{h^{*}}{\sigma}\right) e^{-\frac{s^{2}}{2}} \mathrm{~d} s
$$

En posant :

$$
\begin{gathered}
\bar{S}=\frac{S_{\mathrm{R}}}{S_{\mathrm{N}}} \\
S_{\mathrm{H}}=S_{\mathrm{N}}(1-\bar{S})
\end{gathered}
$$

L'équation (13) devient

$$
F_{\mathrm{EHD}}=\tau_{0} \cdot S_{\mathrm{N}}(1-\bar{S}) \operatorname{ArcSinh}\left(\frac{\eta_{0} \cdot V_{\mathrm{g}}}{h_{\mathrm{c}} \tau_{0}}\right)
$$

Écrivons l'équation du film d'huile en régime élastohydrodynamique d'après Johnson [20]

$$
h_{(x)}=h_{0}+\frac{x^{2}}{2 R_{\mathrm{e}}}+\delta_{(y)}
$$

avec $: \delta_{(y)}=\frac{b^{2}}{2 R_{\mathrm{e}}}$

L'équation de Hertz pour le contact linéique nous donne $b^{2}=\frac{4 W R_{\mathrm{e}}}{\pi l E^{*}}$

L'équation du film au centre dans le cas d'un contact linéaire s'écrit d'après Dowson [5] :

$$
\widehat{H}_{\mathrm{c}}=3,06(\widehat{U})^{0,69}(\widehat{G})^{0,56}(\widehat{W})^{-0,10}
$$

Avec : $\widehat{H}_{\mathrm{c}}=\left(\frac{h_{\mathrm{c}}}{R_{\mathrm{e}}}\right) ; \widehat{U}=\left(\frac{\eta_{0} U}{E^{*} R_{\mathrm{e}}}\right) \widehat{G}=\left(\alpha E^{*}\right)$; $\widehat{W}=\left(\frac{W_{\mathrm{EHD}}}{E^{*} l R_{\mathrm{e}}}\right)$

Au centre du contact, pour $x=0$, l'équation (20) s'écrit :

$$
h_{\mathrm{c}}=h_{0}+\delta_{(y)}
$$

Passons aux paramètres adimensionnels en divisant les membres de l'équation (22) par $R_{\mathrm{e}}$

On peut écrire :

$$
\begin{gathered}
H_{0}=\frac{h_{0}}{R_{\mathrm{e}}}, \\
\Delta=\frac{\delta_{(y)}}{R_{\mathrm{e}}}=\frac{b^{2}}{2 R_{\mathrm{e}}^{2}}=\frac{2}{\pi} \widehat{W}
\end{gathered}
$$

L'équation (22) devient :

$$
\widehat{H}_{\mathrm{C}}=H_{0}+\Delta
$$

d'où

$$
H_{0}=\widehat{H}_{\mathrm{C}}-\Delta
$$

Écrivons : $H_{\mathrm{e}}=3,06(\widehat{U})^{0,69}(\widehat{G})^{0,56}$

L'équation (21) devient :

$$
\widehat{H}_{\mathrm{c}}=H_{\mathrm{e}}(\widehat{W})^{-0,10}
$$

L'équation (24) devient :

$$
H_{\mathrm{e}}(\widehat{W})^{-0,10}-0,63 \widehat{W}-H_{0}=0
$$

La résolution de cette équation nécessite la connaissance de la hauteur initiale $H_{0}$, qui n'est jamais donnée dans les études ayant trait au sujet. Selon Paffoni [14], on la détermine de la manière suivante : à charge élevée on détermine la hauteur du film au centre par l'équation (21), l'écrasement $\Delta$ par l'équation (23) on détermine la valeur de $H_{0}$ par l'équation (25).

L'équation (27) ne possède pas de solution analytique mais se résoud numériquement.

Une fois la valeur de $\widehat{W}$ calculée, elle est introduite dans l'équation (9) pour déterminer $W_{\mathrm{S}}$ tel que :

$$
W_{\mathrm{S}}=W-W_{\mathrm{EHD}}
$$

Le coefficient de frottement de l'équation (11) devient :

$$
f=\frac{f_{\mathrm{S}} \cdot W_{\mathrm{S}}+\tau_{0} \cdot(1-\bar{S}) \operatorname{ArcSinh}\left(\frac{\eta_{0} \cdot V_{\mathrm{g}}}{h_{\mathrm{c}} \tau_{0}}\right)}{W}
$$

Avec $f_{\mathrm{S}}=0,13$ qui représente le frottement en lubrification limite pris comme valeur de référence par L. Qiang [10] et E. Gelink [13]. On l'utilisera dans la suite du calcul pour ce modèle.

\subsection{Résultats et discussion}

Les résultats de calcul du coefficient de frottement pour les quatre lubrifiants en fonction de la vitesse de glissement sont indiqués sur la figure 5 et la variation du coefficient de frottement en fonction du paramètre $\mathrm{S}$ est indiquée sur la figure 6 ; les caractéristiques géométriques du pion et micro-géométriques des surfaces (pions et disques) 


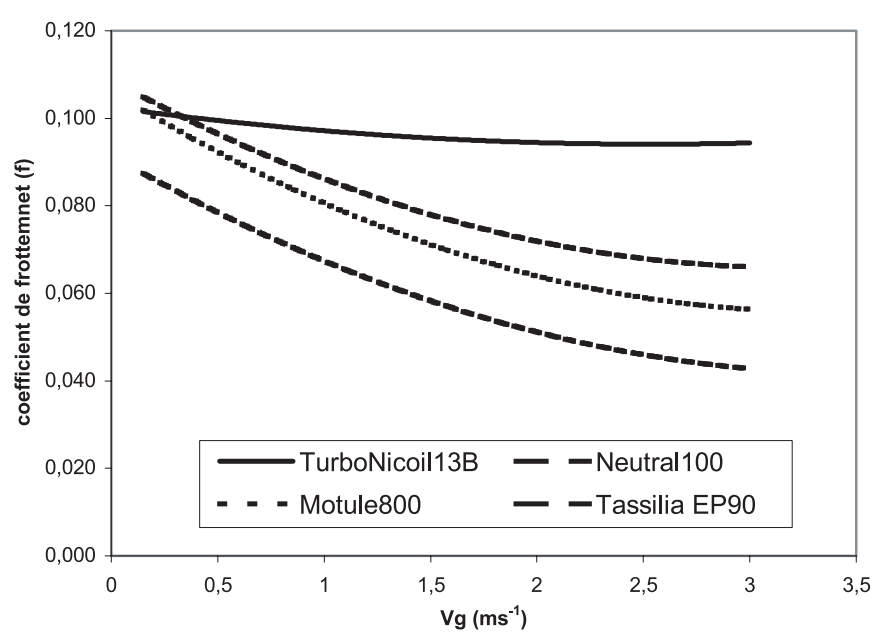

Fig. 5. Variation du coefficient de frottement en fonction de la vitesse de glissement pour $167 \mathrm{MPa}$.

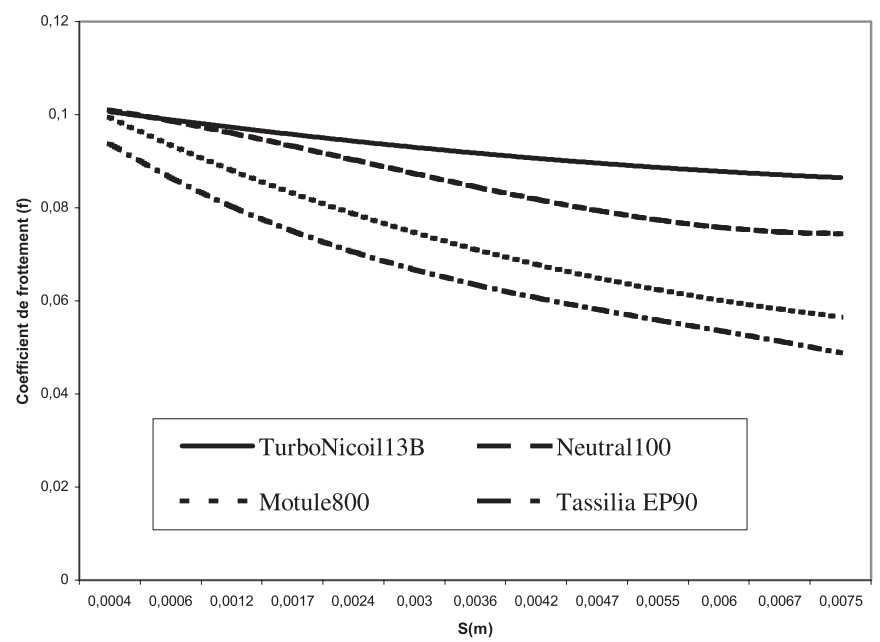

Fig. 6. Variation du coefficient de frottement en fonction de $S$.

Tableau 1. Caractéristiques géométriques et microgéométriques.

\begin{tabular}{|c|c|c|c|c|c|c|c|}
\hline \multicolumn{4}{|c|}{ géométrie du pion } & \multirow[t]{2}{*}{ Module de Young } & \multicolumn{3}{|c|}{$\begin{array}{l}\text { Micro géométrie des } \\
\text { pions et des disques }\end{array}$} \\
\hline$R_{\mathrm{e}}$ & & $l$ & $L$ & & $R_{\mathrm{a}}$ & $n$ & $\beta$ \\
\hline $\mathrm{mm}$ & & $\mathrm{mm}$ & $\mathrm{mm}$ & $231 \mathrm{GPa}$ & $10^{-6} \mathrm{~m}$ & $10^{9} \mathrm{~m}^{-2}$ & $10^{-6} \mathrm{~m}$ \\
\hline 4,7 & 6 & 6 & 8 & & 0,201 & 11 & 25,2 \\
\hline
\end{tabular}

sont indiquées dans le tableau 1 et les caractéristiques des lubrifiants sont données dans le tableau 2. On remarque l'influence convergente d'un certain nombre de paramètres sur la diminution du coefficient de frottement, à savoir :

- la diminution du coefficient de frottement en fonction de la croissance de la vitesse,

- la diminution du coefficient de frottement avec la croissance de la viscosité,

- la diminution du coefficient de frottement avec la croissance du paramètre $\mathrm{S}$

\section{4 Étude expérimentale}

Cette étude consiste à faire des essais de frottement lubrifié simulant le contact linéique sur un tribomètre pion disque, en utilisant quatre lubrifiants pour une gamme des vitesses variant de 0,15 à $3 \mathrm{~m} . \mathrm{s}^{-1}$.

\subsection{Le tribomètre}

La machine est un tribomètre de type pion disque, avec un pion qui est fixe supportant la charge et un disque mobile en rotation, le tout est immergé dans une cuve contenant le lubrifiant à étudier; la photo de la figure 7a présente une vue générale du tribomètre; le détail de la zone encadrée est représenté sur la figure 7b qui illustre le principe de l'essai. Le tribomètre est conçu pour travailler sur différentes gammes de vitesses, allant de 0,03 jusqu'à $10 \mathrm{~m} . \mathrm{s}^{-1}$. Pour nos essais nous avons utilisé la gamme des vitesses de 0,15 à $3 \mathrm{~m} . \mathrm{s}^{-1}$. L'erreur de lecture sur le coefficient de frottement est de l'ordre de $6 \%$.

\subsection{Les éprouvettes}

Le contact à étudier est un contact linéaire hertzien avec des pions et des disques en acier pour construction des engrenages sans traitement thermique. La géométrie des pions a été adaptée pour avoir une forme qui permette l'étude du contact cylindre sur plan comme indiquée par la figure 8. Le rayon de courbure est calculé pour un module métrique ISO $m=6 \mathrm{~mm}$. Les dimensions du pion ont été étudiées pour minimiser l'erreur de calcul sur la vitesse de glissement, qui est inférieure à $5 \%$; les différentes caractéristiques géométrique et micro-géométrique sont celles du tableau 1.

Une précaution particulière est à prendre pour le réglage de l'orthogonalité du montage entre le pion et le disque comme indiqué par les figures $9 \mathrm{a}$ et $\mathrm{b}$.

\subsection{Préparation des essais}

Les essais ont été préparés de la manière suivante.

\subsubsection{Mesures avant essai}

Un certain nombre de mesures ont été effectuées avant les essais :

- la pesée des pions, sur une balance électronique avec une précision de $10^{-4} \mathrm{~g}$;

- la mesure des états de surfaces, des paramètres de Greenwood $(n, \beta)$ des pions et des disques sur un profilomètre (surfas scan $3 \mathrm{~S}$ ),

- la mesure du rayon de courbure des pions sur le même profilomètre. La figure 10 donne un des profils mesurés. 
Tableau 2. Caractéristiques des lubrifiants et synthèse des résultats expérimentaux.

\begin{tabular}{|c|c|c|c|c|c|c|}
\hline \multirow[b]{2}{*}{ Lubrifiants } & \multicolumn{3}{|c|}{ Caractéristiques des lubrifiants } & \multicolumn{3}{|c|}{ Pressions utilisées } \\
\hline & $\begin{array}{c}\text { Viscosités } \\
(\text { Pa.s) }\end{array}$ & $\begin{array}{c}\tau_{0} \\
(\mathrm{MPa})\end{array}$ & $\begin{array}{c}\alpha \\
\left(\mathrm{Pa}^{-1}\right)\end{array}$ & $\begin{array}{c}130 \\
(\mathrm{MPa})\end{array}$ & $\begin{array}{c}167 \\
(\mathrm{MPa})\end{array}$ & $\begin{array}{c}334 \\
(\mathrm{MPa})\end{array}$ \\
\hline Turbo Nicoil 13B & 0,017 & \multirow{4}{*}{2,5} & \multirow{4}{*}{$19 \times 10^{-9}$} & ++ & ++ & - \\
\hline Neutral 100 & 0,029 & & & ++ & ++ & - \\
\hline Motule 800 & 0,154 & & & ++ & ++ & ++ \\
\hline Tassilia EP90 & 0,324 & & & ++ & ++ & ++ \\
\hline
\end{tabular}

++ : Essais qui se sont déroulés sans usure.

- : Essais où il y a eu usure et grippage des pions à cette pression.

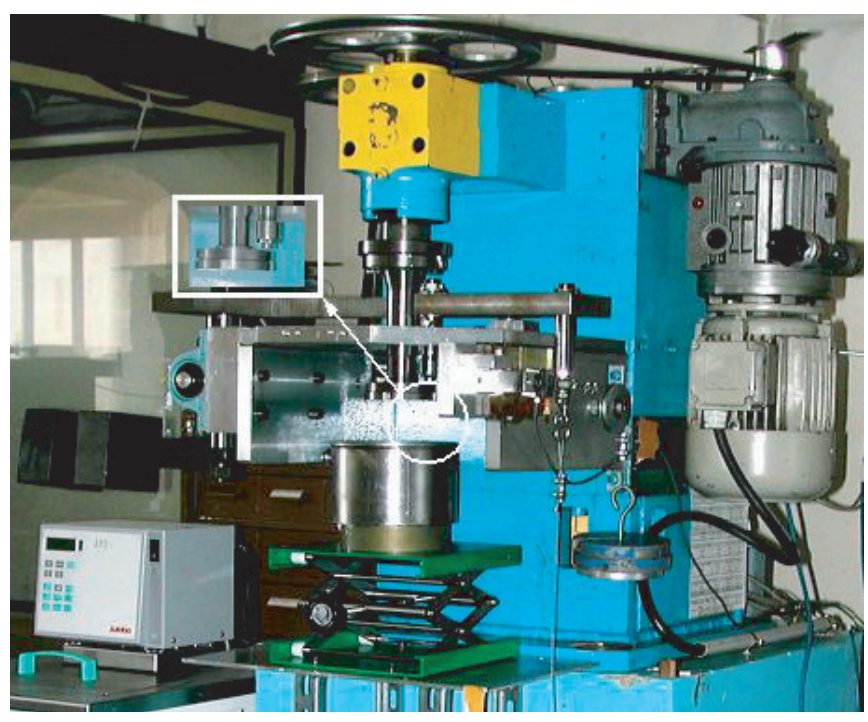

(a)

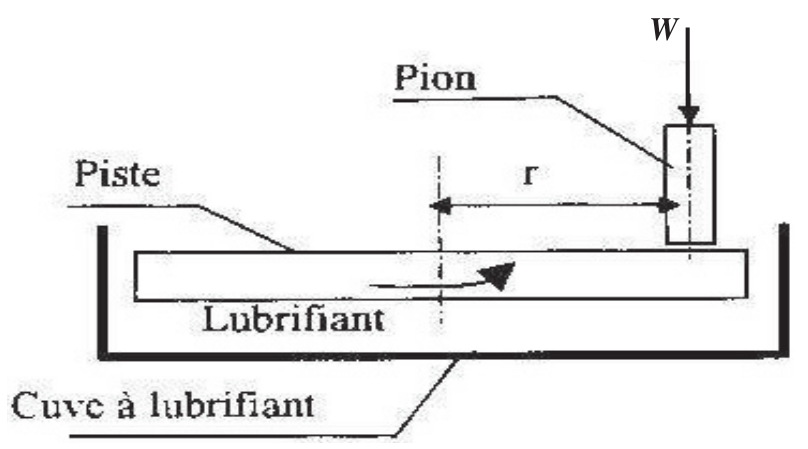

(b)

Fig. 7. a) Vue génerale du tribomètre, b) principe de l'essai pion disque.

\subsubsection{Mise au point d'une procédure}

Les essais ont été effectués à une température ambiante de $20{ }^{\circ} \mathrm{C}$; pour un lubrifiant et une pression fixés, ils consistent à faire varier les vitesses, d'une manière décroissante, puis croissante avec treize paliers avec un temps de maintien par palier $\Delta t$ égal à $20 \mathrm{~s}$; ce temps a été déterminé après un certain nombre d'essais préliminaires pour garantir un minimum de fluctuation de
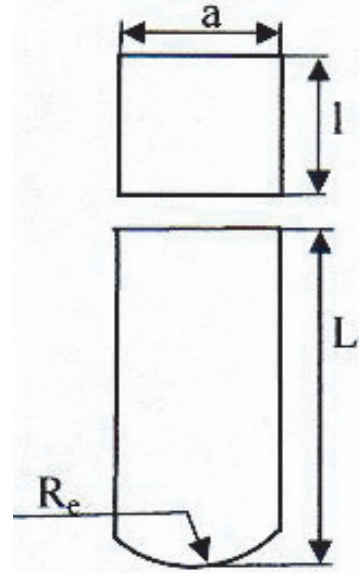

Fig. 8. La géométrie du pion.

la tête de lecture et la stabilité du frottement pour chaque palier. Le diagramme de la figure 11, indique la procédure d'essai. Notre étude s'est limitée à une vitesse maximale de $3 \mathrm{~m} . \mathrm{s}^{-1}$ à cause de la naissance de vibrations au-delà de cette vitesse. Cette gamme de vitesses varie de

$$
V_{\min }=0,15 \mathrm{~m} \cdot \mathrm{s}^{-1} \text { à } V_{\mathrm{Max}}=3 \mathrm{~m} \cdot \mathrm{s}^{-1}
$$

\subsection{Conduite des essais}

Les essais ont été menés de la manière suivante :

- utilisation de quatre lubrifiants de différentes viscosités ;

- utilisation de trois pressions pour chaque lubrifiant;

- utilisation d'une gamme de vitesses variant de 0,15 à $3 \mathrm{~m} . \mathrm{s}^{-1}$ pour tous les lubrifiants.

\section{Résultats et discussions}

Après les essais on a effectué un certain nombre de mesures pour vérifier l'état des pions et des pistes. Pour cela, on a mesuré les états de surfaces des deux éprouvettes et en particulier le profil des pions; entre autres une pesée de ces derniers a été effectuée. On a obtenu les résultats suivants. 


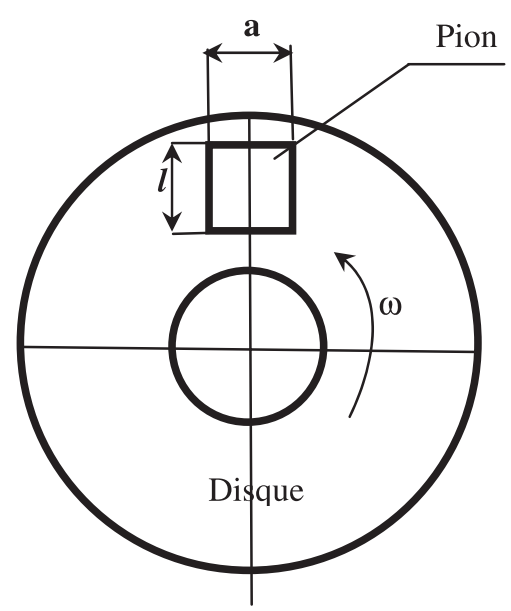

(a)

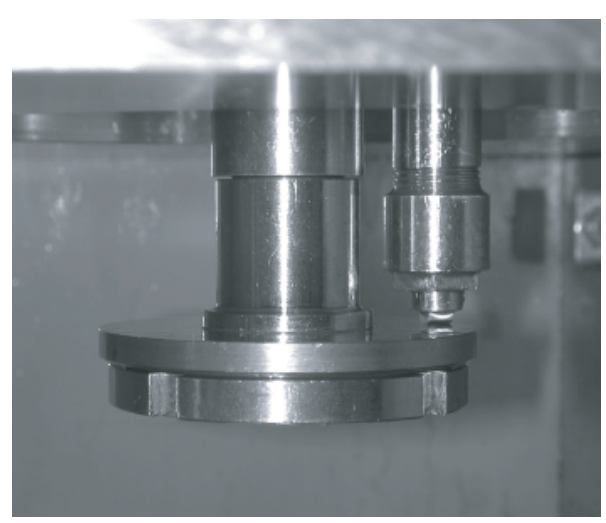

(b)

Fig. 9. a) Disposition du pion par rapport au disque sur la machine. b) Détail de la disposition du pion par rapport au disque en vue de dessus.

\subsection{Résultats}

Ces résultats sont synthétisés dans le tableau 2.

- L'observation des pions ayant subi un grippage montre un changement de forme du pion visible sur la photo de la figure 12 (grossissement 32) et une perte de matière de l'ordre de $3 \%$ par rapport à la masse initiale.

- Pour les pions n'ayant pas subi de grippage, il n'y a pas eu de changement ni sur la forme ni sur la géométrie ou de perte de masse, figure 13.

- Le dépouillement des résultats nous permet de tracer la variation du coefficient de frottement en fonction des vitesses de glissement pour différents lubrifiants figure 14 , ainsi que sa variation en fonction du paramètre $\mathrm{S}$ figure 15 .

\subsection{Discussion}

On remarque après nos essais et le dépouillement des résultats que la variation du coefficient de frottement dépend des trois paramètres que nous avons fait varier, à savoir la vitesse, la viscosité et la pression.

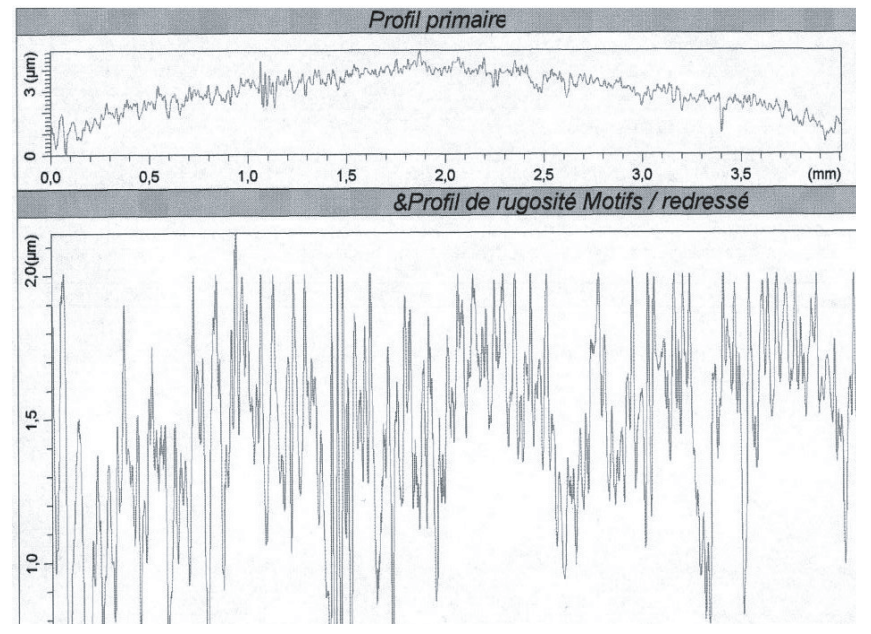

Fig. 10. Profil mesuré.

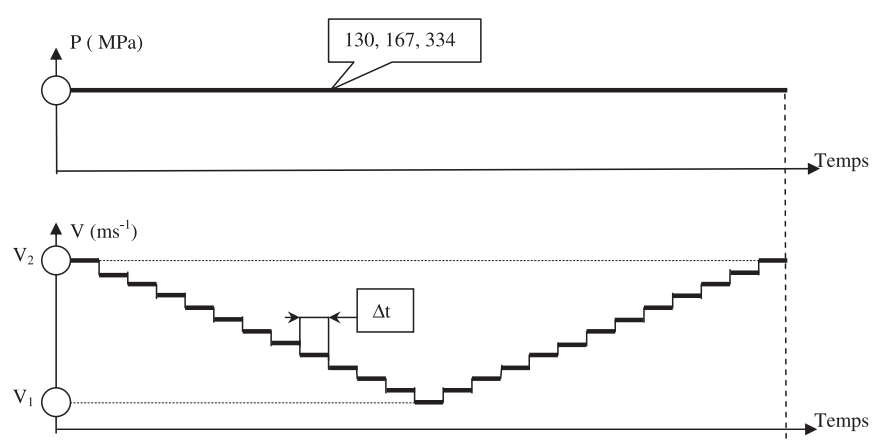

Fig. 11. Procédure expérimentale pour les différents lubrifiants pour une pression fixe et des vitesses variables.

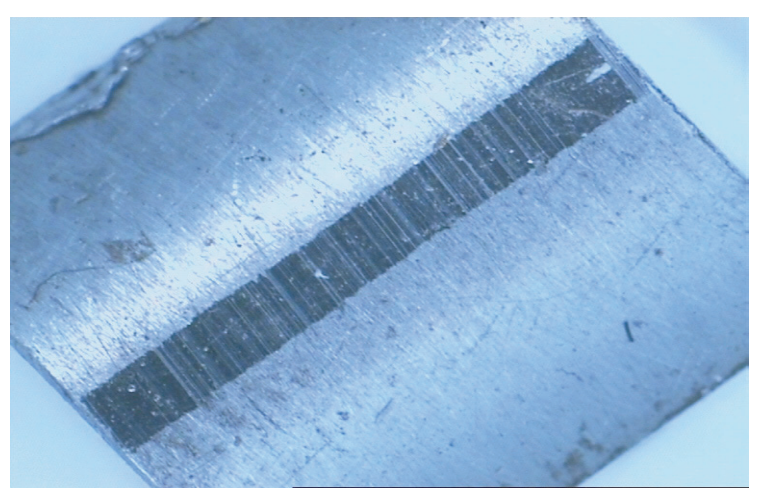

Fig. 12. Photo du comportement du pion pour Turbo Nicoil13B à $334 \mathrm{MPa}$.

- Influence de la vitesse :

Les courbes de la figure 14 nous indiquent que, quel que soit le lubrifiant utilisé, une diminution du coefficient de frottement apparaît avec la croissance de la vitesse de glissement. Les valeurs des coefficients de frottement ne diminuent pas de la même manière quand on passe d'un lubrifiant à un autre ou d'une pression à une autre. 


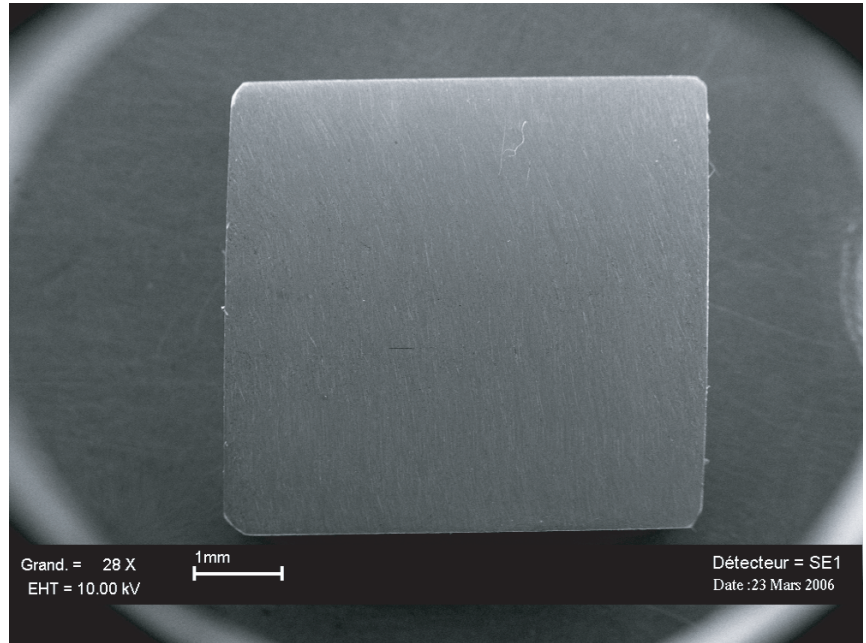

Fig. 13. Pion après essais sans usure.

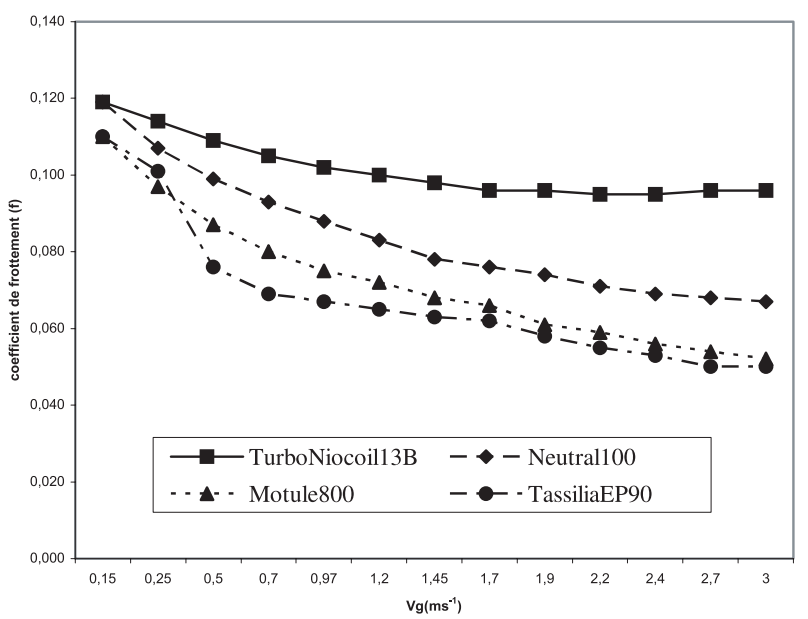

Fig. 14. Courbes de frottement en fonction des vitesses de glissement pour $167 \mathrm{MPa}$.

- Influence de la viscosité :

On remarque que, quelle que soit la pression qui a été utilisée, on a une diminution du coefficient de frottement avec la croissance de la viscosité.

- Influence du nombre $S$ :

Les courbes sur la figure 15 représentent les courbes moyennes de la variation du coefficient de frottement en fonction du paramètre $\mathrm{S}$ des différents essais pour les différents lubrifiants aux pressions et aux vitesses utilisés comme indiqué par le tableau 2.

On remarque que lorsque le paramètre $\mathrm{S}$ croît on a une diminution du coefficient de frottement; entre autres on a remarqué après le dépouillement des résultats pour les différents lubrifiants, qu'on avait une similarité ou de faibles variations entre les valeurs du frottement avec la pression de $167 \mathrm{MPa}$, même à forte pression à $334 \mathrm{MPa}$ pour la Motule 800 et la Tassilia EP90. Par contre pour la Neutral 100 on a eu de grandes différences entre les valeurs obtenues à la pression de $130 \mathrm{MPa}$ et celles obtenues à

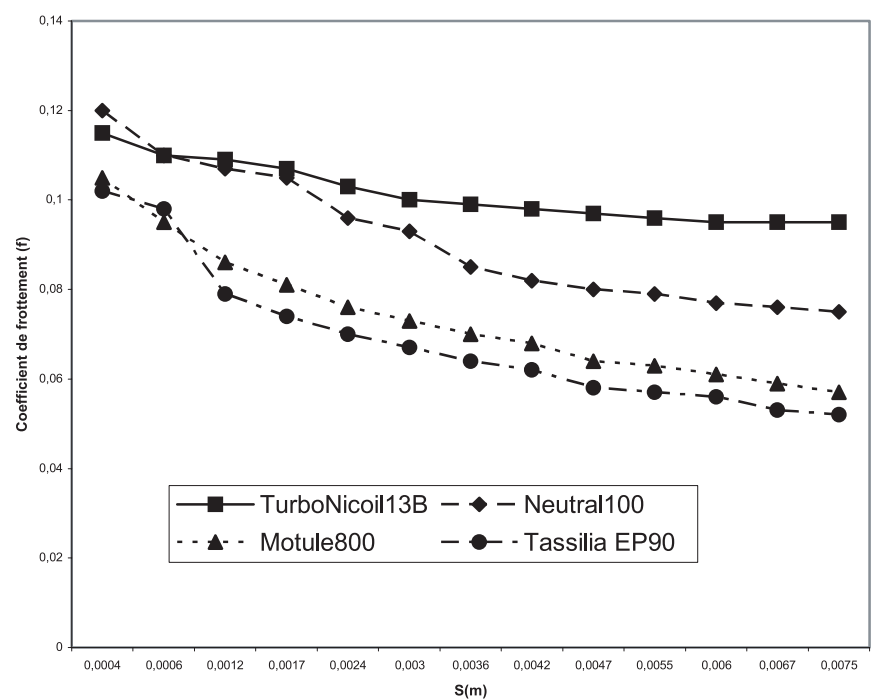

Fig. 15. Courbes de frottement expérimentales pour les différents lubrifiants en fonction du paramètre $\mathrm{S}$.

$167 \mathrm{MPa}$, d'où la variation notable entre la courbe de cette huile figure 14 et celle de la figure 15 .

\section{Confrontation}

En superposant les courbes obtenues à partir du modèle en figure 6 et les courbes expérimentales en figure 15 nous obtenons la figure 16 qui associe les deux résultats. En tenant compte dans notre analyse d'une marge d'erreur de $5 \%$ entre les courbes modélisées et expérimentales, on remarque :

- TurboNicoil13B : La courbe expérimentale est audessus de la courbe modélisée, on note que les deux courbes sont presque linéaires, les valeurs des deux courbes ne se joignent pas mais sont à l'intérieur de la marge des $5 \%$ d'erreur.

- Neutral 100 : La courbe expérimentale se détache par rapport à la courbe modélisée pour les six premières valeurs; les courbes se rejoignent à partir de $S=0,0036$.

- Motule 800 : La première valeur expérimentale se détache de la courbe modélisée, par la suite les deux courbes se rejoignent presque totalement à partir de $S=0,0006$.

- Tassilia EP90 : Les deux premières valeurs de la courbe expérimentale se détachent de la courbe modélisée et par la suite les deux courbes se rejoignent presque totalement à partir de $S=0,0012$.

\section{Discussion}

L'analyse de la confrontation modèle/expérimentation pour les différents lubrifiants nous indique que l'évolution des courbes calculées et obtenues expérimentalement sont comparables, à savoir que le coefficient de frottement diminue avec la croissance de la vitesse, de la viscosité et de $S$. On remarque que la concordance entre les courbes 


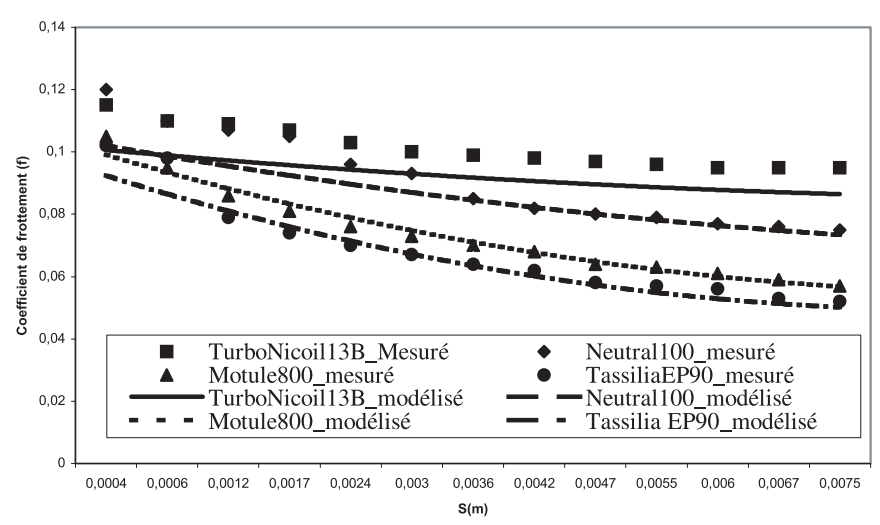

Fig. 16. Confrontation entre les courbes mesurées et celles déterminées par le modèle.

calculées et obtenues expérimentalement croît avec la croissance de la viscosité; c'est le cas de l'huile Motule 800 et Tassilia EP90 d'où leur bon comportement en contact linéaire hertzien.

\section{Conclusion}

Cette étude a atteint un certain nombre d'objectifs, à savoir la simulation expérimentale d'un contact linéique pour une gamme de vitesses variant de 0,15 à $3 \mathrm{~m} . \mathrm{s}^{-1}$, débouchant sur le tracé des courbes de frottement en fonction du paramètre $S$, et leur confrontation avec un modèle théorique. Cette confrontation nous a permis de connaître le comportement de chaque huile testée vis-àvis du contact linéaire en milieu lubrifié.

On remarque que le facteur viscosité a une influence considérable dans les conditions isothermes, car pour la TurboNicoil13B et la Neutral 100 dont les viscosités sont les plus faibles, on a eu apparition du grippage pour une pression moyenne de $334 \mathrm{MPa}$, ce qui n'est pas le cas pour la Motule 800 et la Tassilia EP90 de viscosité plus élevée. Entre autres, on remarque pour une même valeur de $S$ que la convergence entre les valeurs calculées et mesurées augmente avec la croissance de la viscosité.

Les résultats encourageants de cette étude montrent que les moyens expérimentaux et le protocole que nous avons établi sont pertinents. Il est à remarquer que ces résultats concernent une situation dans un état stationnaire de glissement, et on sait que le fonctionnement des engrenages est un contact transitoire d'un point à l'autre sur toute la longueur de contact; nous avons donc prévu une seconde partie qui consiste à calculer la variation du coefficient de frottement le long du contact en connaissant les valeurs extrêmes déterminées expérimentalement et qui fera l'objet d'une future publication. Entre autres nous prévoyons dans une phase ultérieure d'étudier en conditions isothermes la variation du coefficient de frottement en fonction des matériaux et de la variation des états de surfaces en nous appuyant sur les travaux de Nogueira [15] et de mettre au point un modèle adapté au contact linéaire hertzien lubrifié en conditions extrêmes de frottement.
Remerciements. Les auteurs tiennent à remercier l'ensemble du groupe tribologie pour leur soutien à cette étude et les fructueuses discussions sur le sujet.

\section{Références}

[1] R. Lefèvre, Graissage et tribotechnique, Tome 2 engrenages et transmissions, Technip 1975

[2] G. Henriot, Traité théorique et pratique des engrenages Tome 1, Dunod, Paris, 1968

[3] H.M. Martin, The lubrication of gear teeth, Engineering London 102 (1916) 119-121

[4] A.N. Grubin, I.E. Vinogradova, Fundamentals of the hydrodynamic theory of lubrication of heavily loaded cylindrical surfaces, Tsentral. Nauk. Issledovatel Inst. Tekhnol. Mashinostroen, Vol. 30, Moscou, D.S.I.R. London Translation, $\mathrm{n}^{\circ} 337,1949$

[5] D. Dowson, G.R. Higginson, The fundamentals of roller gear lubrication Elastohydrodynamic lubrication, Pergamon Press, London, 1966

[6] X. Jiang, D.Y. Hua, H.S. Cheng, X. Ali, Lee Si. C, Mixed elastohydrodynamic lubrication model with asperity contact, J. Tribology 121 (1999) 481-491

[7] K.L. Johnson, J. Greenwood A, S.Y. Poon, A simple theory of asperity contact in elastohydrodynamic lubrication, Wear 19 (1992) 91-108

[8] N. Patir, H.S. Cheng, An average flow model for determining effects of three dimensional roughness on partial lubrication, ASME J. Lubrication Technology 100 (1978) $12-17$

[9] J.A. Greenwwood, J.P.B. Williamson, Contact of nominally flat surfaces, Phil. Trans. Roy. Soc. London. Ser. A. 295 (1966) 300-319

[10] L. Qiang, Friction in mixed EHD lubricated contact including thermal effect, Ph.D. thesis, Twente University, NL, Septembre 2002

[11] F. Robbe-Valloire, Statistical analysis of asperity on a rough surface, Wear 249 (2001) 401-408

[12] F. Robbe-Valloire, R. Progri, B. Paffoni, Prise en compte de la géométrie dans un contact, communication interne du groupe tribologie, SUPMECA, Paris, décembre 2003.

[13] E. Gelink, Mixed lubrication of line contact, Ph.D. thesis, Twente University, NL, 1999

[14] B. Paffoni, F. Robbe-Valloire, R. Progri, J. Blouet, Frottement iso, pièzo et élastohydrodynamique dans un contact rugueux. Étude théorique et expérimentale, $\mathrm{XV}^{\mathrm{e}}$ congrès Français de Mécanique, Nancy, 2001

[15] I. Nogueira, An experimental model for mixed friction during running, Wear 253 (2002) 541-549

[16] Bulletin $\mathrm{N}^{\circ} 95$, Institut de l'engrenage et des transmissions, octobre 1989

[17] V.N. Borsoff, M. Godet, A scoring factor of gears, ASLE Transaction, 2, April 1963

[18] J.I. Mc Cool, Comparison of models for the contact of rough surfaces, Wear 107 (1986) 37-60

[19] J.C. Bell, J.W.Kannel, C.M. Allen, The rheological behaviour of the lubricant in the contact zone of a rolling contact system, ASME, J. Basic Eng., 86 (1964) 423

[20] K.L. Johnson, Contact mechanics, Cambridge University press, 1996 